\title{
Fault Diagnosis based on Semi-supervised Global LSSVM for Analog Circuit
}

\author{
Chen Chen \\ College of Engineering \\ Bohai University \\ Jinzhou, China \\ 934194434@qq.com
}

\author{
Aihua Zhang \\ College of Engineering \\ Bohai University \\ Jinzhou, China \\ Jsxinxi_zah@163.com
}

\begin{abstract}
Aiming at the analog circuit performance online evaluation demand of the largest interval principle and underlying geometric structure, two online methods of dimension reduction are proposed for analog circuit performance evaluation from the angle of feature extraction, First, a supervised method of dimension reduction based on Fisher's Linear Discriminant Analysis (LDA) is presented to increase the classification distance largely. This method is a well-known scheme for feature extraction and dimension reduction. However, the incomplete classification will lead to great influence on performance evaluation accuracy. Based on this, another feature extraction strategy by Locality Preserving Projections (LPP) is proposed. LPP should be seen as an alternative unsupervised approach to Principal Component Analysis (PCA). This method properly obtains a local space that best detects the essential manifold structure. In this paper, the fault diagnosis can be recognized via the Global and Local Preserving based Semi-supervised Support Vector Machine (semi-supervised Global LSS VM). The experiment takes a typical Sallen-key low-pass circuit as diagnosis object. In order to prove the effectiveness of the proposed method in this paper, the traditional fault diagnosis method based on standard support vector machine (S VM) is employed also. The diagnosis speed and accuracy are all proved via numerical simulation.
\end{abstract}

Keywords-Analog Circuit; Faults Diagnosis; Semisupervised; LDA; LPP

\section{INTRODUCTION}

Analog circuit fault diagnosis and performance evaluation are currently the most important blocks in every real-world system ${ }^{[1]}$. Under the circumstance of increased integration and comple xity in large-scale integrated circuit, the operation reliability has been improved. However, the issues such as availability of the poor fault models, component tolerances and circuit nonlinearities are the typical bottleneck in the circuit testing leading to slow evolution in the fault diagnosis processes ${ }^{[2]}$. The requirement of real-world could be hindered if the traditional analog circuit fault diagnosis methods like parameter identification method, expert system, fault dictionary et al. ${ }^{[3]}$ are still used. Artificial intelligence like wavelet transform, neural network, fuzzy theory and support vector machine (SVM) methods are put forward with scientific and technological progress to meet realistic demands. In this work, wavelet analysis and fuzzy theory can be considered as two crucial methodologies in fault diagnosis system. Last but not least, SVM outstrips these two in modeling. Quite a few studies focus on SVM not only in classification application but regression. The primary advantage of this approach is that simple structure, global optimum and strong generalization ability can be exhibited when solving small sample, nonlinear and high dimensional pattern recognition problems. However, this method will lead to insufficiency in learning only with scarcely labeled samples. Meanwhile, the lack of es sential information and structure of data will result in great influence on the performance evaluation accuracy.

In order to solve the insufficiency problem above, some experts put their eyes on unsupervised learning, namely only utilize unlabeled patterns in huge quantities and applied them in analog circu it fault diagnosis research. $\mathrm{Li}$ et al. [4]presented a novel unsupervised learning method for human action categories. This work considers diversity of human action categories, and selects multiple sets of movements such as skating, walking et al, so it reveals the sufficiency of data utilization. However, the drawback largely comes from uncertainty. Focusing on this issue, Reference [5] proposed a novel method to detect which method was proper, unsupervised learning or supervised method. The experiment demonstrates that the unsupervised learning would not require a laborious labeling process, a clear forerunner for practical purposes if unknown attacks can be expected. Li et al [6]e mployed a method to choose samples with strong characteristics, and thus decreased the unknown attacks in unsupervised learning process.

Although the unsupervised learning method is in excess of the supervision mechanism on generalization performance, it is more difficult to solve interfere problem. As is well known, most interference or noise comes from the uncertainty. Many researchers put forward methods based on semi-supervised learning to realize circuit fault diagnosis, namely, a training set consisting of labeled and unlabeled patterns. This method balances between supervised and unsupervised ideas, and also makes full use of the data resource under improved generalization ability. Zhang et al. [7] proposed a novel semi-supervised Traffic Identification based on Affinity Propagation. The method presents the concept of Affinity Propagation which makes running efficient and easy to implement ${ }^{[8]}$. Li et al. [9] introduced the average (mean) into semi-supervised support vector machines (S3VM), and it achieves the biggest interval between mean values of the two classes. From the results, it is clear that this method is much more efficient than traditional SVM, especially on a relatively large data sets (such as more than 1000 samples data sets), and the computation speed has improved significantly. 
Before researchers utilize any classification method, it is beneficial to first perform dimension reduction to project an image into a low dimensional feature space, owing to the consideration of learn-ability and computational efficiency. Specifically, learning from examples is computationally in-feasible if it has to rely on highdimensional representations. Dimension reduction method is commonly used in two aspects ${ }^{[10]}$ : global geometric structure and local information. The global respect such as PCA and LDA are frequently-used. Reference [11] presents that PCA is an unsupervised method based global information and structure; the purpose of this method is to find a mapping subspace on the basis of the minimum mean square error to best represent original data. On the contrary, LDA is a supervised procedure which principle also is to find a mapping subspace; this subspace will obtain the largest distance between within-class and between-class. On the other hand, the local structure would be performed in LPP, Laplacian Eigen-map (LE) and Locality Linear Embedding (LLE) ${ }^{[12]}$.Specially, LPP not only maintains the geometric structure of data set, but also overcomes the defect of other ways in receiving lowdimensional mapping of new samples ${ }^{[13]}$.

The rest of this paper is organized as follows: Section 2 briefly reviews the princip les of the Global LSS VM fault diagnosis system. Section 3 proposes Global LSSVM idea and model. Simulation results are presented in section 4, some conclusions are given in section 5.

\section{FAULT DIAGNOSIS SYSTEM BASED ON GLOBAL LSSVM}

Fault diagnosis systemcontains five parts as follows:

- Analog circuit: $1 \mathrm{kHz}$ Sallen-key low-pass filter is proposed for experiment. For convenience of analysis, all parameters' settings of in this paper are the same as given in literature[14].

- Fault feature extraction: It is the most important step in pattern recognition. In this paper, LDA and LPP are adopted to reduce dimension ${ }^{[8]}$.In addition, algorithm is programmed in LSS VM Toolbox of MatlabR2010bsoftware and the nodal voltages have been simulated by Multisim12.0.

- Fault pretreatment: In order to obtain good classification effect of SVM, the extracted voltages from Multisim12.0 software need to have pretreatment of normalized $[-1,1]^{[15]}$.

- Global LSSVM: Global and local preserving of fault information is concerned for semiunsupervised learning algorithm of analog circuit faults diagnosis, the within-class scatter of LDA combines LPP to fully consider the global and local geometric structure between samples. The process includes two steps: training stage and diagnosis stage. At every stage, samples are all come from pretreatment in step (3).

- Fault diagnosis: Focusing on the single fault of analog circuit, the effectiveness of the proposed approach is finally illustrated by simulations on the Sallen-key low-pass filter circuit ${ }^{[16][16]}$.

And the corres ponding diagram is shown in Fig. 1:

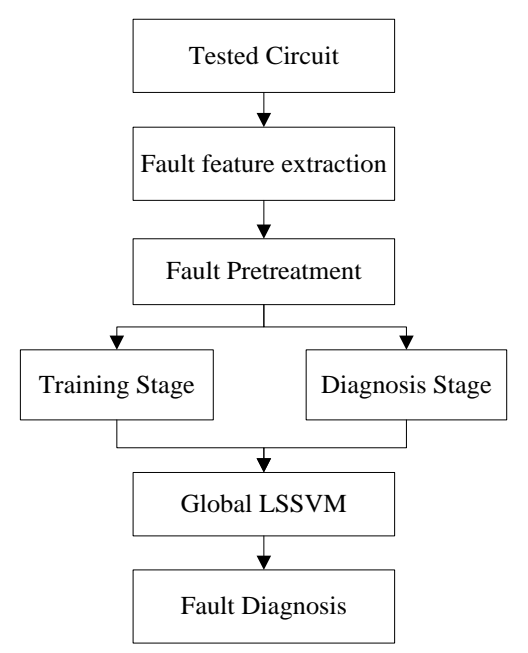

Figure 1. Fault diagnosis system flow diagram

\section{THE FAULT DIAGNOSIS MODEL BASED ON GLOBAL LSSVM}

\section{A. SVM theory description}

SVM has been introduced by Vapnik ${ }^{[17]}$ for solving classification and nonlinear functional estimation problem.

Given a training data set of $m$ points $\left(x_{i}, y_{i}\right)_{i=1}^{m}$ with input data $x_{i} \in \boldsymbol{R}^{n}$ and output data $y_{i} \in \boldsymbol{R}$; The $i$-th element of a vector $\boldsymbol{x}$ is denoted by $x_{i}$, then one feature vector $x_{i}$ with label +1 belongs to class 1 , and the other one with label -1 belongs to class 2 . The quadratic optimization problem of SVM can be transformed as follows

$$
\left\{\begin{array}{l}
\min _{\omega, \xi_{l}}\left\{\frac{1}{2}\left(\omega^{\mathrm{T}} \cdot \omega\right)+H \sum_{i=1}^{m} \xi_{i}\right\} \\
\text { s.t. } y_{i}\left[\left(\omega^{\mathrm{T}} \cdot \varphi\left(x_{i}\right)\right)+b\right] \geq 1-\xi_{i} \\
\xi_{i} \geq 0
\end{array}\right.
$$

It's difficult to solve a quadratic programming (QP) problem. Then, the constrained optimization problem is solved via Lagrangian multipliers under the KarushKuhn-Tucker (KKT) condition

$$
\left\{\begin{aligned}
\max _{\alpha} & \sum_{i=1}^{n} \alpha_{i}-\frac{1}{2} \sum_{i=1}^{n} \sum_{j=1}^{n} \alpha_{i} \alpha_{j} y_{i} y_{j} x_{i} x_{j} \\
\text { s.t. } & 0 \leq \alpha_{i} \leq H, i=1,2, \ldots, m \\
& \sum_{i=1}^{n} \alpha_{i} y_{i}=0
\end{aligned}\right.
$$

where $\alpha_{i}$ denotes Lagrange multiplier.

The decision function for the optimal hyperplane is addressed as follows

$$
f(x)=\operatorname{sgn}\left\{\sum_{i=1}^{n} \alpha_{i}^{*} y_{i}\left(x_{i}, x\right)+b^{*}\right\}
$$

\section{B. Manifold regularization based SVM combined with $L P P(M R-L P P)$}

The traditional regularization based $S_{V M}{ }^{[18]}$ has been widely used in pattern recognition. Through the research 
about regularization, this approach tends to smoothness of classification function, and not concerned about sample information implicitly. Therefore, the geometric structure and information of samples has not been fully applied. Reference [19] introduces the view of manifold learning method to traditional regularization method, so the manifold-regularization framework is addressed as follows

$$
\min _{f \in H_{k}} \frac{1}{l} \sum_{i=1}^{l} V\left(x_{i}, y_{i}, f\left(x_{i}\right)\right)+\gamma_{A}\|f\|_{K}^{2}+\gamma_{I}\|f\|_{I}^{2}(4)
$$

where

$$
V\left(x_{i}, y_{i}, f\left(x_{i}\right)\right)= \begin{cases}0 & y_{i} f\left(x_{i}\right) \geq 1 \\ 1-y_{i} f\left(x_{i}\right) & \text { otherwise }\end{cases}
$$

where $\|f\|_{K}^{2}$ is proposed to control comple xity of controller, $\|f\|_{I}^{2}$ reflects inner manifold structure of samples.

According to (5), combined with LPP, the original optimization problem can be defined by

$$
\begin{array}{cl}
\min _{w \in \boldsymbol{R}^{n}, \xi \in \boldsymbol{R}^{l}} & \frac{1}{l} \sum_{i=1}^{l} \xi_{i}+\gamma_{A}\|w\|^{2}+\gamma_{I} w^{\mathrm{T}} \boldsymbol{X} \boldsymbol{L} \boldsymbol{X}^{\mathrm{T}} w \\
\text { s.t. } & y_{i}\left(\left(w, x_{i}\right)+b\right) \geq 1-\xi_{i}, i=1, \ldots, l
\end{array}
$$

where $\boldsymbol{X}$ consis ts of labled data numbered $l$ and unlabeled data numbered $u . w$ denotes normal vector of hyperplane.

\section{MR-LPP combined with LDA (semi-supervised Global LSSVM)}

From (6), regularization formula $w^{\mathrm{T}} \boldsymbol{X} \boldsymbol{L} \boldsymbol{X}^{\mathrm{T}} w$ only represents local manifold structure, then this section will add global manifold structure to MR-LPP framework. Meanwhile several parameters need to be introduced before applying the algorithm. Laplace matrix $\boldsymbol{L}_{w}$ of adjacency graph $G$ is proposed for nonlinear analog circuit process $^{[20]}$. Meanwhile, the matrix of within class scatter $S_{w}$ is transformed into the form of the graph Laplace.

Labeled set: $\boldsymbol{D}=\left\{x_{1}, \ldots, x_{l}\right\}$

where $\forall x_{i} \in \boldsymbol{R}^{n}$ denotes input value of sample, they respectively belongs to two different categories $\left(C_{+}, C_{-}\right)$, the sample subset $D_{k}$ which size is $l_{k}$ belongs to class $k$. The authority matrix $\boldsymbol{W}$ denotes $k$-neighbor adjacency graph $G$.

$$
\boldsymbol{W}_{i j}=\left\{\begin{array}{lc}
\frac{1}{l_{k}} & \text { if } x_{i} \text { and } x_{j} \text { both belong to class } k \\
0 & \text { otherwise }
\end{array}\right.
$$

Given the normal vector $w$ of classification decision, then $w^{\mathrm{T}} \boldsymbol{S}_{w} w$ denotes within class scatter.

$$
\begin{gathered}
\boldsymbol{S}_{w}=\sum_{k=1}^{2} \sum_{x \in D_{k}}\left(x-u_{k}\right)\left(x-u_{k}\right)^{\mathrm{T}} \\
u_{k}=\frac{1}{l_{k}} \sum_{x \in D_{k}} x(k=1,2)
\end{gathered}
$$

where $\boldsymbol{S}_{w}$ denotes matrix of within class scatter, $u_{k}$ denotes mean value

$$
S_{w}=\boldsymbol{X}_{l} \boldsymbol{L}_{w} \boldsymbol{X}_{l}^{\mathrm{T}}
$$

Equation(10) denotes Laplace matrix of graph corresponding to $S_{w}$.

where $\boldsymbol{L}_{w}=\boldsymbol{I}-\boldsymbol{W}, \boldsymbol{I}$ denotes matrix with one.

Unlabeled set: $\boldsymbol{F}=\left\{x_{l+1}, \ldots, x_{l+u}\right\}$.

where $\boldsymbol{X}_{l+u}=\left\{x_{1}, \ldots, x_{l}, x_{l+1}, \ldots, x_{l+u}\right\}$ includes labeled and unlabeled samples. Among others, $w^{\mathrm{T}} \boldsymbol{S}_{w} w$ denotes within-class scatter corresponding to labeled sample $l, \boldsymbol{L}$ from literature[21]denotes Laplace matrix of adjacency graph corresponding to data set $\boldsymbol{X}_{l+u}$.

Nonlinear Ker - GLSSVM method for the original optimization problem can be transformed by:

$$
\begin{aligned}
& \min _{a, b, \xi} \frac{1}{l} \sum_{i=0}^{l} \xi_{i}+\frac{\gamma_{A}}{2} a^{\mathrm{T}} \overline{\boldsymbol{K}}_{l} \boldsymbol{L}_{w} \overline{\boldsymbol{K}}_{l}^{\mathrm{T}} a+\frac{\gamma_{I}}{2} a^{\mathrm{T}} \overline{\boldsymbol{K}}_{l+u} \boldsymbol{L} \overline{\boldsymbol{K}}_{l+u} a \\
& \text { s.t. } y_{i}\left(\sum_{j=1}^{l+u} a_{j} \boldsymbol{K}\left(x_{i}, x_{j}\right)+b\right) \geq 1-\xi_{i}, i=1, \ldots, l
\end{aligned}
$$

where $\boldsymbol{K}($,$) denotes Mercer - kernel function.$

For illustrative purpose, the nonlinear Ker-GLSSVM flow chart can be expressed as:

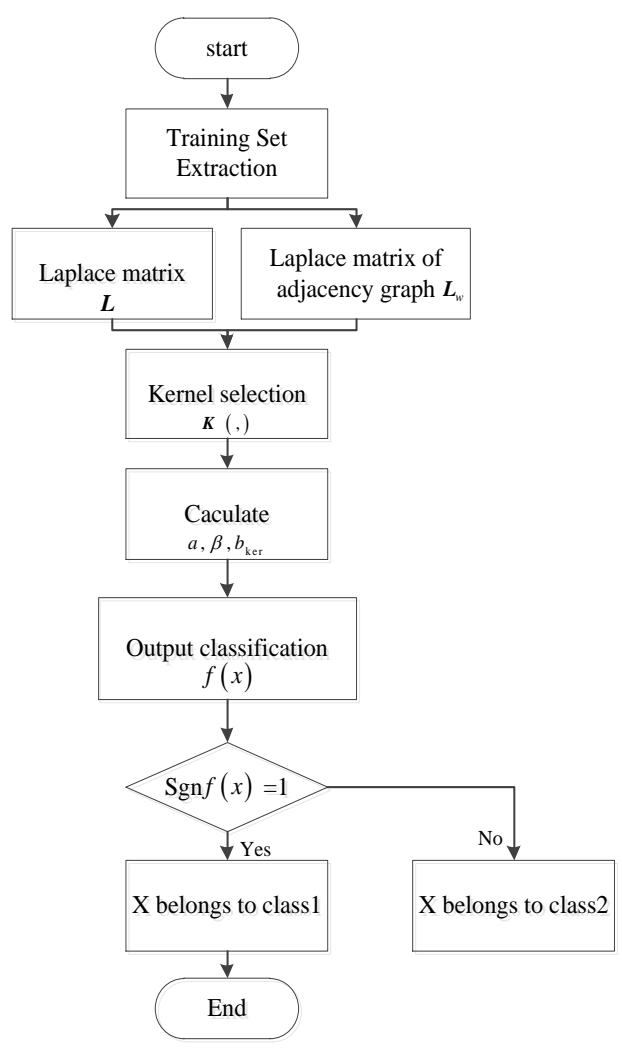

Figure 2. Nonlinear Ker-GLSSVM flow chart 


\section{EXPERIMENT}

\section{A. Diagnostic Circuit and Fault Type}

Experiment adopts an normal analog circuit Sallen-key low-pass filter as the diagnostic object shown in Fig. 3.

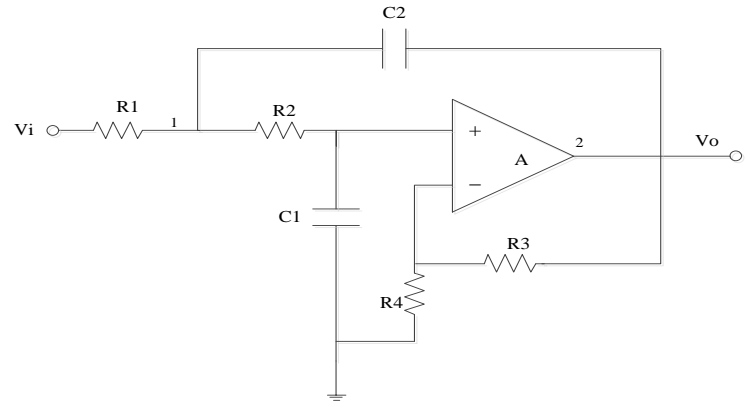

$(\mathrm{R} 1=\mathrm{R} 2=15.8 \mathrm{k} \Omega, \mathrm{R} 3=10 \mathrm{k} \Omega, \mathrm{R} 4=17.8 \mathrm{k} \Omega$,

$\mathrm{C} 1=\mathrm{C} 2=10 \mathrm{nF}$ )

Figure 3. $1 \mathrm{kHz}$ Sallen-key low-pass filter
A Sallen-key low-pass filter circuit showed in Fig. 3 is used as the exemplary circuit. The nominal value of each component is also labeled in Fig. 3. Operation circuit consists of four resistances R1, R2, R3, R4; two capacitances $\mathrm{C} 1, \mathrm{C} 2$ and amplifier $\mathrm{A}$. This analog circuit adopts RC network with positive feedback structure to perform low-pass filter. The (+) and (-) represents the parametric variation in the component value $+50 \%$ and $50 \%$ respectively. If the parameter perturbation of each element is beyond its tolerance range, it is thought to be faulty. For the convenience of analysis ${ }^{[22]}$, only considers single soft faults from part of the capacitance and resistance which can be divided into two modes: (+) or (-) represents the fault value is upward bias or downward bias the component normal value. Total fault set can be defined as shown in Tab.1.

TABLE I. FAULT TYPE, CODE AND VALUE

\begin{tabular}{cccccccccc}
\hline Fault Type & R2 $(+)$ & R2 $(-)$ & R3 $(+)$ & R3(-) & C1(+) & C1(-) & C2(+) & C2(-) \\
\hline Fault Code & F1 & F2 & F3 & F4 & F5 & F6 & F7 & F8 \\
Fault Value & $47.4 \mathrm{k}$ & $7.11 \mathrm{k}$ & $30 \mathrm{k}$ & $4.5 \mathrm{k}$ & $30 \mathrm{nF}$ & $4.5 \mathrm{nF}$ & $30 \mathrm{nF}$ & $4.5 \mathrm{nF}$ \\
\hline
\end{tabular}

\section{B. Numerical experiments}

In this part, researchers conducted experiments with a total of 60 datasets: 20 datas ets for training and 40 for test. For convenience of analysis, all datasets were normalized to be in $[-1,1]$ from normalization and all settings of parameters in this paper are the same as that given in literature[22]. Additionally, researchers utilize LSSVM Toolbox to help programming in the platform of Matlab R2010b software. The nodal voltages have been simulated by Multisim12.0. During simulation process, when fault components in mode $(+)$, its value is 3.0 times of nominal while fault components in mode (-) is 0.45 times of nominal value. The tolerance of any resistance and capacitance is assumed as $50 \%$ of nominal value. Applying sine excitation signal of $20 \mathrm{~V}, 1.0 \mathrm{kHz}$ and according to topology of the circuit, two testing nodes are applied, which are numbered nodes land 2.

To validate the effectiveness of the proposed semisupervised Global LSSVM in this paper, the traditional fault diagnosis method based on SVM proposed in literature [23] is also employed for the comparison purpose while the analog circuit fault diagnosis is on. In this section, test data number is 40 . In the procession, it contains two data sets, plus sign and hollow circle respectively represent two different categories. Classification performs in the two-dimensional plane ${ }^{[24]}$, so the separating hyperplane is a line. Fig. 5 shows that classification effect is better than that of Fig. 4.

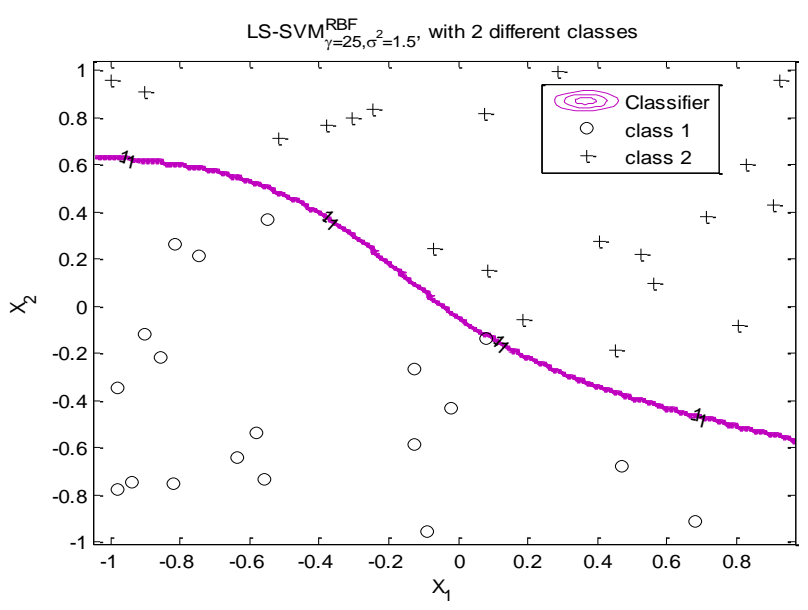

Figure 4. Classification of SVM

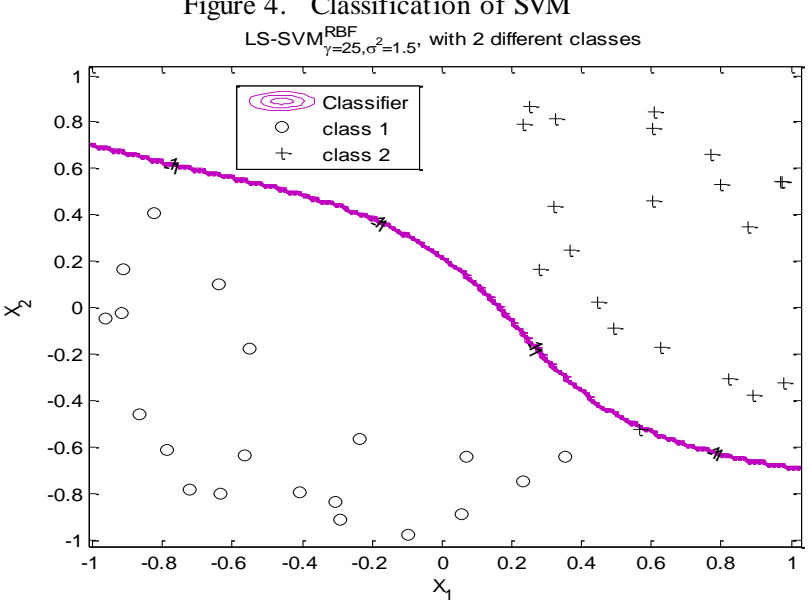

Figure 5. Classification of Global LSSVM

Experimental results above only have shown the diagnosis effect between two different categories, the computational time between semi-supervised Global 
LSSVM and traditional SVM is revealed in Tab.2. It is obviously that the proposed strategy is very satisfied not only the time respond ability but also the fault diagnosis accuracy[25].
Given corresponding contrast in speed and accuracy of two methods in the process of operation:

TABLE II. COMPARISON BETWEEN SVM AND SEMI-SUPERVISED GLOBAL LSSVM

\begin{tabular}{ccccc}
\hline \multirow{2}{*}{ Evaluation Algorithm } & \multicolumn{3}{c}{ Evaluation Indexes } \\
\cline { 2 - 5 } SVM & Training & Testing & Class & Accuracy \\
\hline Semi-supervised Global LSSVM & 20 & 40 & 2 & $78.45 \%$ \\
& 20 & 40 & 2 & $75.25 \%$ \\
\hline
\end{tabular}

\section{CONCLUSIONS}

In this paper, two novel online dimensionality reductions LDA and LPP are proposed in traditional SVM to find underlying geometric structures between samples and increase the sufficiency of learning. LDA is capable of obtaining the largest distance of global geometric structure between within-class and between-class but local structure is largely ignored; the proposal LPP is proved to achieve local geometric structure. Simulation results have shown that semi-supervised Global LSSVM is an attractive solution to balancing the geometric structure and sufficiency of learning. In engineering practice, considering the high operation rate and evaluation precision, this strategy is worth to be applied and developed. In addition, this approach is proven to be valuable in the diagnosis of multi-faults in analog circuit, which will be the future research problem.

\section{ACKNOWLEDGMENT}

The present work was supported partially by the National Natural Science Foundation of China (Project no: 61304149) and Natural Science Foundation of Liaoning, China (Project no: 2013020044).The authors highly appreciate the above financial support.

\section{REFERENCES}

[1] X. Zhang D. Qiu, and F. Chen, "Support vector machine with parameter optimization by a novel hybrid method and its application to fault diagnosis," Neurocomputing, vol. 149, pp. 641651,2015 .

[2] P. Bilski, "Analysis of the classifier fusion efficiency in the diagnostics of the accelerometer," Measurement, vol. 67, pp. 116$125,2015$.

[3] J. Shi, W. An, and L. Qiao, "Application of fault dictionary diagnosis methods in the testability field," Prognostics and System Health Management Conference (PHM-2014 Hunan), IEEE, pp. 218-222, 2014.

[4] F. Li, H. Wang, C. Juan et al., "Unsupervised Learning of Human Action Categories Using Spatial-Temporal Words," Int J Comput Vis, vol. 79,pp. 299-318,2008.

[5] Pavel Laskov, Konrad Rjeck et al., "Learning intrusion detection: supervised or unsupervised," Computer Engineering and Application, no. 14,pp. 74-77, 2004.

[6] X. Li, J. Liu, and Z. Shi, “A Chinese WebPage Classifier based on support vector machines and unsupervised clustering," Chinese J. Computers, vol.24, no. 1,pp.62-68, 2001

[7] Z. Zhang, B. Wang, X. Li et al., "Semi-supervised Traffic Identification based on Affinity Propagation," Automatica Sinica , vol. 38 , no. 1, pp. 1-10,2012.

[8] Y.-F. Li, J. T. Kwok, and Z.-H. Zhou, "Semi-supervised learning using label mean," pp. 633-640, 2009
[9] L. Yang, and L. Wang, "A class of semi-supervised support vector machines by DC programming" Analysis and Classification, vol. 7, no. 4, pp. 417-433,2013.

[10] X. He, S. Yan, Y.Hu et al., "Learning a Locality Preserving Subspace for Visual Recognition" IEEE Intemational Conference on, vol. 2, no. 6,pp. 1449-1459, 2003

[11] G. Chen, S E. Qian, “ Denoising of hyperspectral imagery using principal component analysis and wavelet shrinkage," Geoscience and Remote Sensing, IEEE Transactions on, vol. 49, no.3, pp. 973980, 2011.

[12] W. S. Gou J, Deng Z. "Global and local preserving based on semisupervised support vector machine," ACTA ELECTRONIC SINCA, vol.7, no.7, pp.1626-1633, 2010.

[13] H. Wang, S. Chen, Z. Hu, and W. Zheng "Locality-preserved maximum information projection," IEEE Transactions on Neural Networks, vol. 19, no.4,pp.571-585, 2008.

[14] Y. Wang. C. Li, L. Wang. "Fault diagnosis in analog circuit based on PCA and SVM," Joumal of Electronic Measurement and Instrument, vol. 22, no.3, pp.64-69, 2008.

[15] R M. Sargis, B A. Neel, C O. Brock, et al., “ The novel endocrine disruptor tolylfluanid impairs insulin signaling in primary rodent and human adipocytes through a reduction in insulin receptor substrate-1 levels," Biochimica et Biophysica Acta (BBA)Molecular Basis of Disease, vol.1822, no.6, pp.952-960, 2012.

[16] S. Yin, X. Yang, and H. R. Karimi. "Data-driven adaptive observer for fault diagnosis," Mathematical Problems in Engineering, pp.832-836, 2012.

[17] V. Vapnik, The nature of statistical leaming theory: Springer, 2000.

[18] C. Zhang and Haykin S. "On different facets of regularization theory" Neural Computer, vol. 14, no. 12, pp. 2791-2846, 2002.

[19] Belkin M, Niyogi P and Sindhwani V. "Manifold regularization: geometric framework for learning from examples", Journal of Machine Learning Research, no. 7, pp. 2399-2434, 2006.

[20] S. Ding S. Yin, K. Peng, H. Hao, and B. Shen. "A novel scheme for key performance indicator prediction and diagnosis with application to an industrial hot strip mill," Industrial Informatics, IEEE Transactions on, vol. 9, no.4, pp.2239-2247, 2012.

[21] X. He, S. Yan, Y. Hu, P. Niyogi, and H.-J. Zhang. "Face recognition using Laplacianfaces," IEEE Transactions on Pattern Analysis and Machine Intelligence, vol. 27, no.3, pp.328-340, 2005.

[22] S. Yin, X. Yang, and H. R. Karimi, "Data-driven adaptive observer for fault diagnosis," Mathematical Problems in Engineering, vol.2012,2012.

[23] A. Zhang, X. Huo, and Z. zhang, "Strategy for evaluation of analog circuit performance based on adaptive and it erat ive LSSVR with multi RBF kernel tuning," Journal of Electronic Measurement and Instrument, vol. 27, no. 2, pp. 115-119, 2013.

[24] Y J. Zhang, J J. Hou, L. Huang, "Fault Diagnosis of Analog Circuits with Tolerance Based on Simulation-before-Test and Distance Algorithm," Applied Mechanics and Materials, pp. 475: 156-160, 2014.

[25] S. Y. Gou K, Zhu Y. "Advance in modern analog circuit intelligent fault diagnosis methods," Electronic design engineering, vol. 20, no.2,pp.177-180, 2012. 